\title{
Envelope instability and the fourth order resonance
}

\author{
Chao $\mathrm{Li}^{*}$ and Ya Liang Zhao \\ Key Laboratory of Particle Acceleration Physics and Technology, Institute of High Energy Physics, \\ Chinese Academy of Sciences, 19(B) Yuquan Road, Beijing 100049, People's Republic of China
}

(Received 21 September 2014; published 19 December 2014)

\begin{abstract}
The well-known envelope instability or the second order even collective mode [I. Hofmann, Phys. Rev. E 57, 4 (1998)] and the fourth order resonance $4 \sigma=360^{\circ}$ due to the nonlinear space charge effect in high intensity beams have been studied previously. A wide stop band around $15^{\circ}$ is found in a pure periodic focusing channel. In addition, it is illustrated that the fourth order resonance dominates over the envelope instability and practically replaces it in the stop band [D. Jeon et al., Phys. Rev. ST Accel. Beams 12, 054204 (2009)]. In this paper, for a continuous beam with remarkable space charge, our 2D self-consistent particle-in-cell simulation work with the code TOPOPIC shows these two kinds of effects respectively in a periodic focusing defocusing (FD) channel. For a fixed tune depression $\eta=0.8$, a stop band with a width of almost $15^{\circ}$ is also demonstrated. Moreover, it is confirmed that analytical results of the rms envelope instability diagram are a valid tool to interpret the width of the stop band. Emittance growth rates in stop band are also well explained. It is found that, for a nearly rms matched beam, the emittance growth in the stop band is almost proportional to the saturation time of the nonlinear instability of the envelope, which happens in a quick manner and takes only a few FD cells. In contrast, the fourth order resonance is independent of rms matching and will be accompanied by beam evolution as "a long term effect" once the related mechanism is excited.
\end{abstract}

DOI: 10.1103/PhysRevSTAB.17.124202

PACS numbers: 41.75.-i, 29.27.Bd, 29.20.Ej

\section{INTRODUCTION}

The role of space charge has generated adequate attention in recent years due to the increasing interest in highpower linear as well as circular accelerators [1-3]. In this subject, great efforts are focused on space-charge-induced nonlinear resonances [4]. An abundance of work has been launched in the past several years, such as particle-core resonance [5,6], the well-known envelope instability $[7,8]$, and coherent collective nonlinear resonances $[9,10]$, etc. Resonance phenomena related to the nonlinear space charge normally will deteriorate beam quality, cause beam collective instability, and lead to beam halo formation and beam loss, which oppose the utmost goal in accelerator design and operation [11]. Consequently, both of the effects caused by collective modes of the beam and the single particle lattice nonlinearities must be considered to understand the problems of resonance crossing [12] and halo formation [11]. Unfortunately, these space-charge-related effects are usually mixed and reciprocal because of the beam self-consistency, which makes the analytical analysis of the whole system nearly insurmountable.

The bulk of this study is dedicated to a comprehensive discussion on the fourth order resonance $4 \sigma=360^{\circ}$, which

\footnotetext{
*lichao@ihep.ac.cn

Published by the American Physical Society under the terms of the Creative Commons Attribution 3.0 License. Further distribution of this work must maintain attribution to the author $(s)$ and the published article's title, journal citation, and DOI.
}

is the equivalent of $4 \nu=1$ in a circular accelerator and the well-known envelope instability in a periodic focusing defocusing (FD) channel with appreciable space charge. The space charge effect on a beam close to the space charge limit $\eta<0.2$, where all of the resonance stop bands are completely overlapped and the approach to equipartition [13] may be a universal feature, is beyond the topic studied in this paper. In earlier research launched by Jeon [14], a wide stop band around $15^{\circ}$ is found in a pure period focusing channel. In the stop band, it stated that the fourth order resonance dominates over the envelope instability and practically replaces it. In 2009, the stop band width of about $20^{\circ}$ of the fourth order $4 \sigma=360^{\circ}$ was first experimentally detected at GSI UNILAC, and perfect consistency has been obtained between measurement and simulation [15]. The contribution of the presented work is a reaffirmation of the stop band near $4 \sigma=360^{\circ}$. Moreover, clear analytical interpretations of the stop band width and the "sharp falling effect" in the direction of decreasing phase advance $\sigma$ are given. For a systematic study when the conditions of both envelope instability and the fourth order of resonance are satisfied, the time scales of the effectiveness of envelope instability and the fourth order resonance are also discussed. For the case of beam envelope instability, it is found that the saturation $\tau$, which can be considered as the time scale of detuning of the envelope oscillation mode, takes only a few FD periods. Moreover, the saturation time $\tau$ is almost proportional to emittance growth. Whereas, for the case of the fourth order of resonance, the time scale is much longer than the 
saturation time of the beam envelope instability. Once the condition of the fourth order resonance is satisfied, it will be accompanied by the beam evolution.

To aid the reader and to keep the flow of the text clear, we first specify the terminologies "resonance" and "instability" appearing frequently in this paper. Resonance can be simply understood as a certain kind of collision of the eigenvalues, characteristic exponents, or frequencies. In the case when resonance occurs, even small periodic driving forces can produce large amplitude oscillations, which represent a kind of instability. However, a resonance can lead to instability, but not always. Instability occurs for equilibria when the colliding eigenvalues split and go symmetrically in the complex plane [16].

\section{SIMULATION RESULTS AND INTERPRETATION}

\section{A. Physical model and simulation results}

Considering a continuous beam evolution in a pure periodic FD channel, the Hamiltonian for the envelope oscillation is

$$
\begin{aligned}
H_{\mathrm{env}}= & \frac{1}{2}\left(p_{x}^{2}+p_{y}^{2}\right)+V_{\mathrm{env}} \\
= & \frac{1}{2}\left(p_{x}^{2}+p_{y}^{2}+k_{x}(s) \tilde{x}^{2}+k_{y}(s) \tilde{y}^{2}\right) \\
& -1 / 2 K \ln (\tilde{x}+\tilde{y})+\frac{\varepsilon_{x}^{2}}{2 \tilde{x}^{2}}+\frac{\varepsilon_{y}^{2}}{2 \tilde{y}^{2}} .
\end{aligned}
$$

The matched beam envelope can be obtained as the equilibrium solution of the envelope Hamiltonian, which gives

$$
\frac{\partial V_{\mathrm{env}}}{\partial \tilde{x}}=\frac{\partial V_{\mathrm{env}}}{\partial \tilde{y}}=0
$$

With a matched (equilibrium) beam $\tilde{X}, \tilde{Y}$, the phase advance $\sigma_{j}(j=x, y)$, defined as the phase shift of the matched envelope oscillation over one period of the focusing field, is of great importance, which can be evaluated as

$$
\sigma_{j}=\int_{s}^{s+L} \frac{\epsilon_{j}}{\tilde{j}^{2}} d s, \quad j=X, Y .
$$

In the limit of zero space charge $K=0$, it is noted that $\sigma_{j 0}=$ $\lim _{K \rightarrow 0} \sigma_{j}$ as the undepressed phase advance. The term $\eta=\sigma_{j} / \sigma_{j 0}$ is defined as tune depression to measure the importance of the space charge in the related degree of freedom.

For the numerical study, a standard 2D particle-in-cell space charge solver in a rectangular conducting boundary employed in the time domain code TOPOPIC $[17,18]$ is adopted. A nearly rms matched parabolic (PB) initial distribution in the transverse direction is adopted to

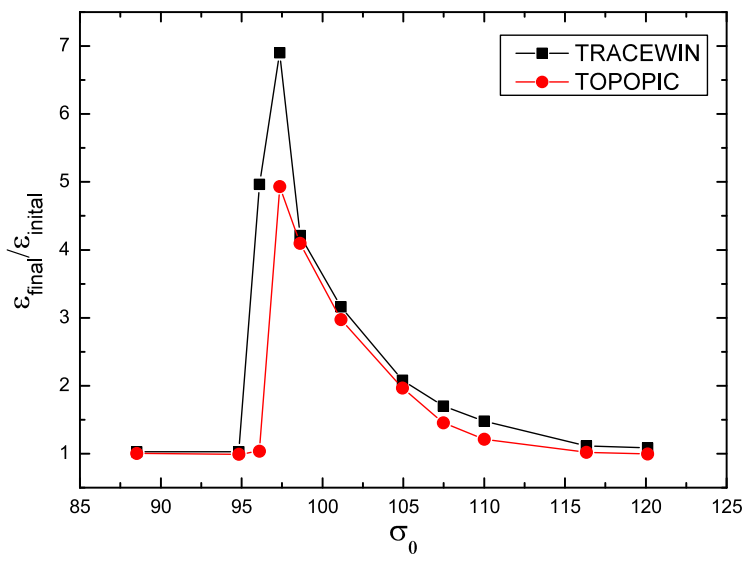

FIG. 1. Simulation results showing the final relative emittance growth for initial parabolic distribution $\left(\eta_{i}=0.8\right)$ with $30 \mathrm{~K}$ macroparticles as functions of zero beam current phase advance $\sigma_{0}$.

represent a continuous proton beam, with the following parameters: rms emittance $\epsilon_{x}=\epsilon_{y}=0.447045$ [ $\mathrm{cm} \mathrm{mrad}$ ] and momentum $\beta \gamma=0.0178818$. A number of $30 \mathrm{~K}$ macroparticles are tracked in the pure FD channel with different depressed phase advances $\sigma$ but with fixed tune depression $\eta_{i}=0.8$. Grids are set as $N x=N y=2^{7}$ for Poisson's equation solver. Figure 1 shows the average rms emittance growth $\left(\epsilon_{x}+\epsilon_{y}\right) / 2$ due to the space charge effect as a function of zero beam current phase advances $\sigma_{0}$. The results from the well-established simulation code TRACEWIN [19] and TOPOPIC show quite good agreement.

Basically, there are two characteristics of the curve in Fig. 1. First, a wide stop band, in which the emittances grow significantly, of almost $15^{\circ}$ is demonstrated, $95^{\circ}<\sigma_{0}<$ $110^{\circ}$. Second, with the phase advance $\sigma_{0}$ decreasing from right to left, the emittance growth rate smoothly goes up to the peak at $\sigma_{0}=97^{\circ}$ and then drops suddenly, called the sharp falling effect here. Both of these characteristics will be discussed in detail in the following sections.

\section{B. Envelope instability stop band}

The properties of collective beam instability have been studied since 1970 [9]. The basic idea is to use the classic linearized perturbation theory to find the eigenmodes of the perturbation of the "Vlasov equation." The envelopeinstability-related space charge was systematically discussed in Ref. [7] and gives the suggestion to the designer that an accelerator should be established in the region where zero beam current phase advance $\sigma_{0}$ is less than $90^{\circ}$. Hofmann derived the generalized formation of stability criterion with a "dispersion relationship" and the envelope instability is also included, noted as the "second order even mode" [8]. In our cases, for a beam in the 2D FD periodic channel, it is mainly the $\nu_{x}-\nu_{y}=0$ resonance that stimulate the envelope instability.

Figure 2 shows the locations of initial working points in the envelope instability diagram [7]. Compared with Fig. 1, 


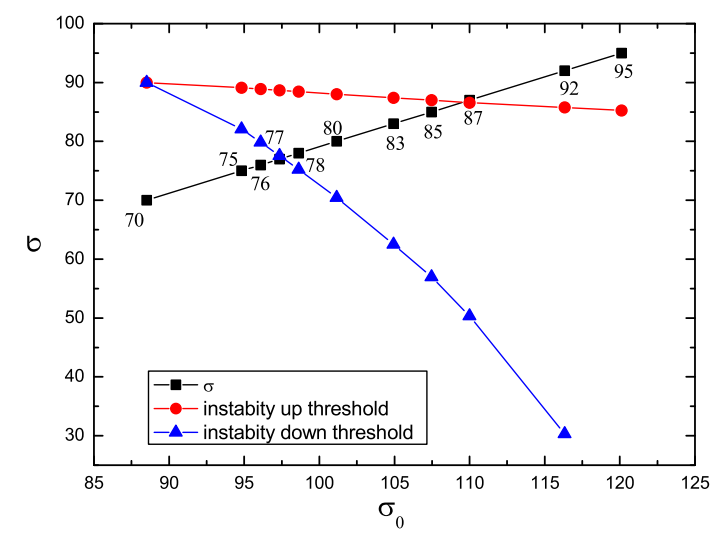

FIG. 2. Instability diagram of the envelope for tune depression $\eta=0.8$. (Red, up threshold; blue, down threshold; black, work points.)

clearly, emittance growth takes place only when working points are located between the red and blue lines, which represent the upper and lower boundaries, respectively, of envelope instability. When working points jump out of the instability diagram in both directions, increasing or decreasing of $\sigma_{0}$, the average emittance does not increase any more. This perfect accordance of the stop band width about $15^{\circ}$ from $95^{\circ}<\sigma_{0}<110^{\circ}$ gives us confidence that the envelope resonance does affect the beam as a collective effect.

As discussed, another characteristic in the stop band is the sharp falling and "smooth falling" of emittance growth rates in directions of increasing and decreasing phase advance around $\sigma_{0}=97^{\circ}$, where $\sigma=77^{\circ}$, at which point the emittance growth is more than $500 \%$. To study this sharp falling effect in one direction, the saturation time $\tau$ for each run to reach a new "equilibrium state" is estimated. It is crucial to note that the equilibrium state means the beam as a whole is saturated with the beam envelope instability but not a real thermal equilibrium state. (What is more, the calculation of saturation is beyond the linearized perturbation theory; the listed following process will show why we call it "estimated.") Take $\sigma=87^{\circ}$, for example; Fig. 3 shows the rms beam size, emittance, and phase advance evolutions as functions of the period number. After about ten periods, which is used here to measure the saturation time for envelope instability in this case, the envelope and emittance get stable and do not have obvious changing. Simultaneously, phase advances of the two degrees of freedom also become equal and stay constant along the channel; in this circumstance, the beam itself is supposed to reach a new equilibrium state with self-adapted or self-detuned parameters as the phase advance and tune depression, noted as $\sigma_{e}$ and $\eta_{e}$, respectively.

Figure 4 shows the changes between initial working points and equilibrium state working points. Clearly, in the envelope instability stop band, all of the beam self-adapted to a new equilibrium state under the influence of the inherent space charge force. Meanwhile, the working points

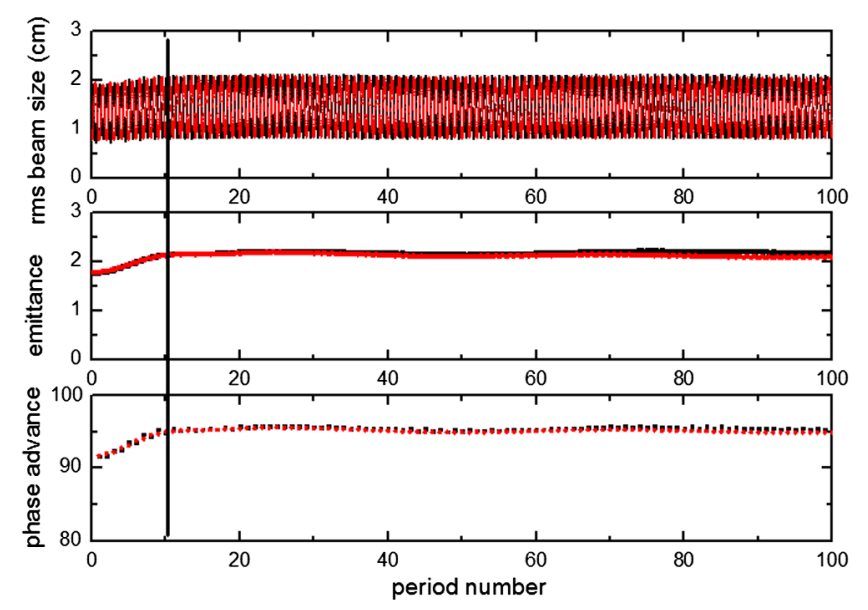

FIG. 3. Plot of the rms beam size, emittance, and phase advance evolutions for $\sigma=87^{\circ}$ with the period number. (Black, $x$ direction; red, $y$ direction.)

are also self-detuned up to the stable region in the direction where the space charge takes a weaker importance, shown by the green dots. It clearly tells us that the envelope instability is, to some degree, an instantaneous or local characteristic, which usually cannot affect the beam as a long term effect because of the self-detuning effect. Obviously, for the case $\sigma_{0}=97^{\circ}$, where $\sigma=77^{\circ}$ in Fig. 4, compared to other work points in the stop band, it has to endure a longer path, which also means a longer saturation time $\tau$ and larger emittance growth to escape from the stop band and reach equilibrium state. Figure 5 shows the emittance growth vs saturation time $\tau$. Not surprisingly, the emittance growth rate is almost proportional to $\tau$. Qualitatively speaking, this curve agrees well with the basic understanding of this process as discussed previously. The related curve between normalized emittance growth and the ratio of tune depression between equilibrium and initial $\eta_{e} / \eta_{i}$ is presented in Fig. 5(b). It can be observed that the growth rate $\epsilon_{e} / \epsilon_{i}$ is almost a quadratic function with $\eta_{e} / \eta_{i}$ as $\epsilon_{e} / \epsilon_{i}=$ $\eta_{e} / \eta_{i}+\eta_{e} \sigma_{i}\left(\eta_{e} / \eta_{i}-1\right)^{2}$.

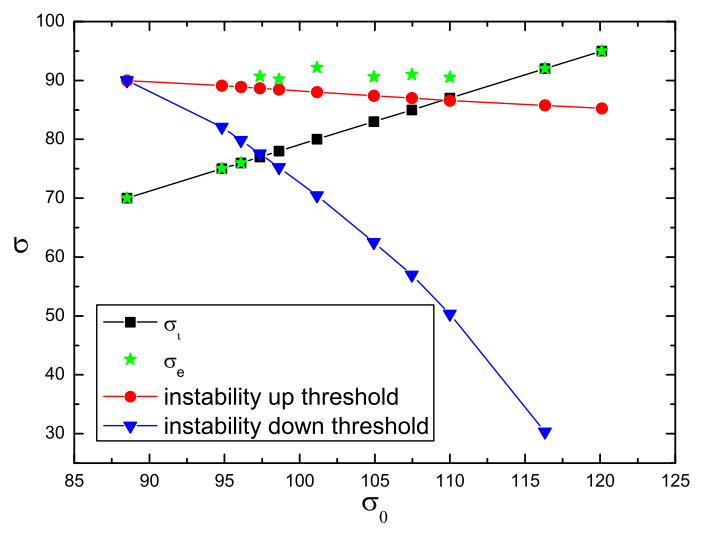

FIG. 4. Plot of initial working points $\sigma_{i}$ (black) and equilibrium state working points $\sigma_{e}$ (green) in the stability diagram for $\eta=0.9$. 


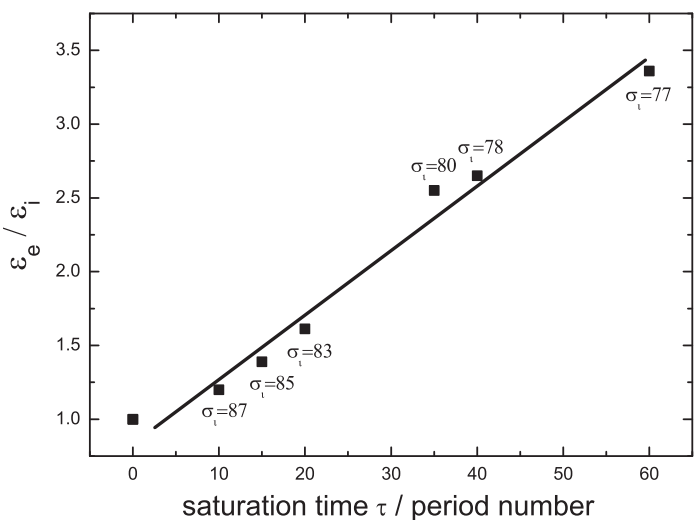

(a)

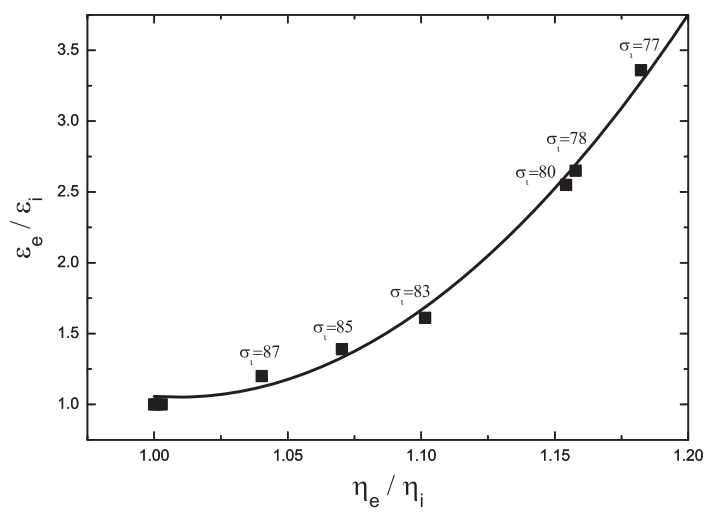

(b)

FIG. 5. Simulation results showing final relative emittance growth for initial parabolic distribution $\left(\eta_{i}=0.8\right)$ with $30 \mathrm{~K}$ macroparticles as functions of (a) saturation time $\tau$ and (b) $\eta_{e} / \eta_{i}$ (down).

The question may be raised why the beam cannot escape from the stop band through the down threshold. The main point is that, under the comprehensive influence of an external field and internal space charge field, if there is any resonance or instability occurs, the beam itself will always try to get to a state where the space charge takes a weaker importance. Thus, with the beam evolving self-consistently, either in an envelope stability stop band or not, the beam always tries to get rid of the force imbalance through the mechanism of emittance or beam halo growth, which can be considered as a kind of "relief" from the energy point of view. The working points in the stop band play a role as a stimulator for the strong nonlinear resonances in a short time range (about ten periods for the case of $\sigma=87^{\circ}$ ). Once this process happens and is accompanied with emittance growth, consequently, in the beam itself, the space charge always takes a weaker importance.

\section{Effect of fourth resonance: $4 \sigma=360^{\circ}$}

The fourth order resonance discussed here is, to some extent, a kind of particle-core resonance. For a beam with nonzero current, the oscillation frequency of a particle is amplitude dependent. Once the resonance condition is
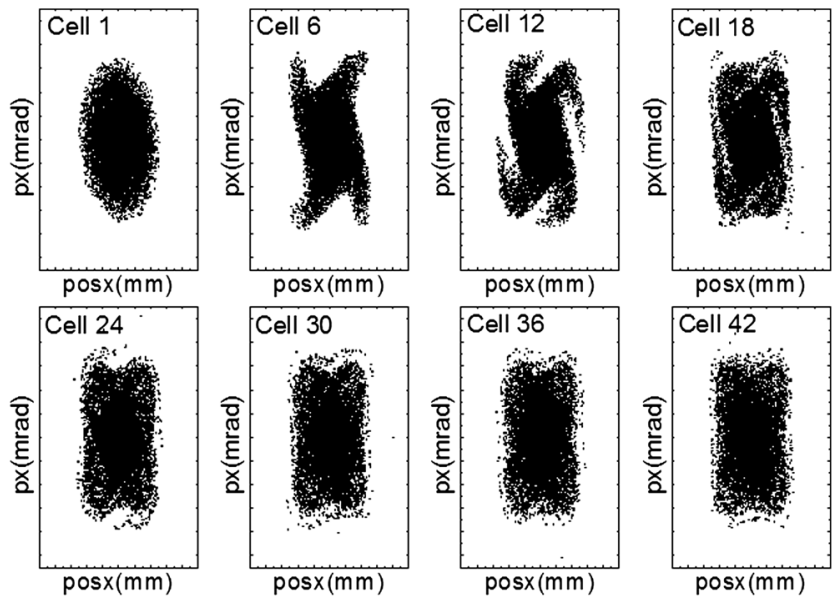

(a)
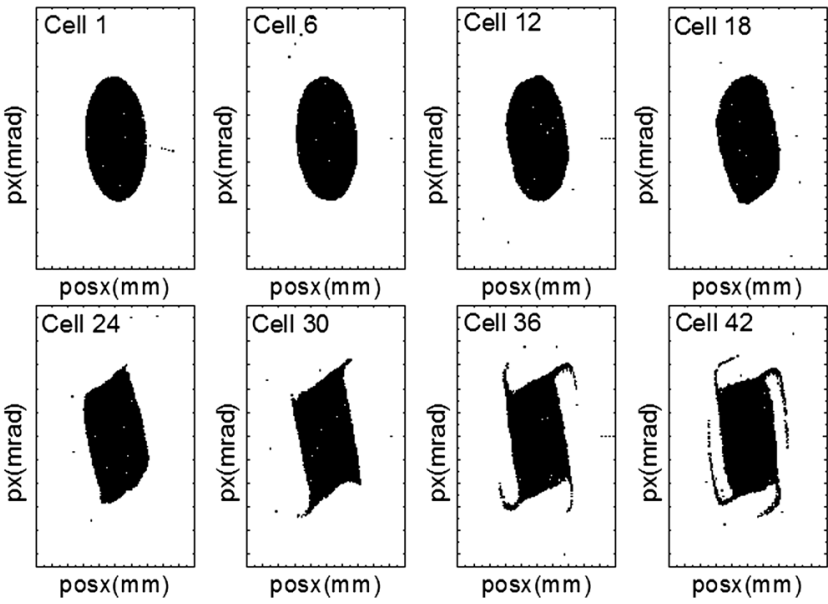

(b)

FIG. 6. Beam profile evolution in $x$ - $p x$ phase space for $\sigma_{i}=87$, $\eta_{i}=0.8$, with (a) parabolic and (b) KV initial distribution. The axis scale is $(-5$ to $5 \mathrm{~cm})$ in the pos $x$ direction and $(-125$ to $125 \mathrm{mrad})$ in the $p x$ direction.

satisfied, particles always have more possibility to be captured by and trapped in the fourth order resonance. It is important to point out that the fourth order resonance is also excited in the stop band discussed above. Figure 6 shows the beam profile evolution, with $\mathrm{rms}$ matched parabolic and Kapchinskij-Vladimirskij (KV) initial distribution in $x$ - $p x$ phase space, when $\sigma=87^{\circ}$. Compared with the KV case, the fourfold structure in phase space formed much clearer and quicker for the parabolic distribution in the first several FD cells. This is mainly because there are some particles initially trapped in the fourth order resonance islands with matched $\mathrm{PB}$ distribution; however, for the nearly rms matched $\mathrm{KV}$ beam (numerical noise contained), it takes a much longer time for particles to be captured by and trapped in resonance, here almost 40 periods. In both cases, with the beam evolution, halo particles appear accompanied by emittance growth. Information of ten test particles uniformly located on the 


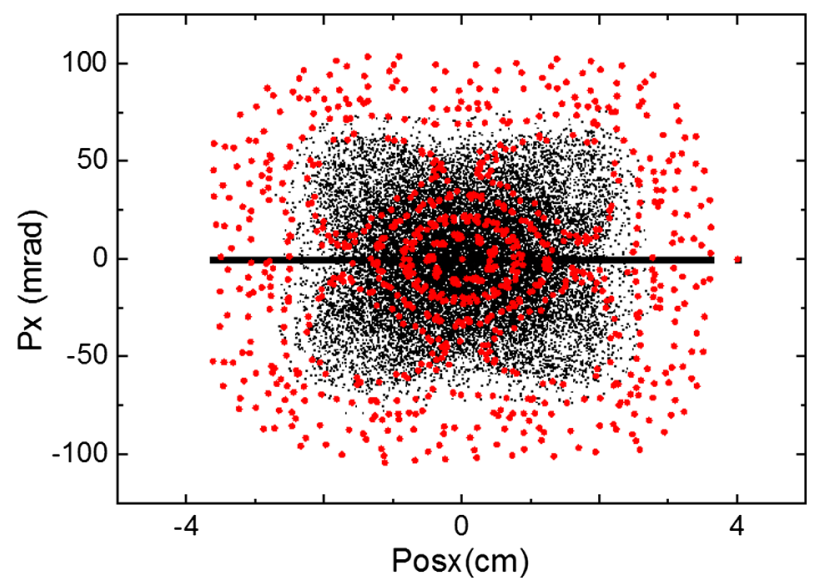

FIG. 7. Plots of beam particle distribution in phase space at cell 100 (black dots) and Poincaré surface plot of ten test particles over 100 FD periods.

$x$ axis are recorded at the end of each FD period over 100 periods to get the Poincare surface plot. The red dots in Fig. 7 show the resonance structure of the system, which agrees well with the phase map, represented by the black dots, at cell 100.

It can be observed that the fourth order resonance is a long term effect. In a periodic FD channel, once this resonance condition is satisfied, particles captured by, trapped in, and escaping from the four stable islands will be accompanied by the beam evolution from the first moment, and this is one of the main sources of beam halo besides rms match conditions. The trapping condition is formulated in the term of an adiabaticity parameter $T=\frac{\partial x_{f}}{\partial n} \frac{1}{Q_{x f(n)} \Delta x(n)}$ [20]. Moreover, this resonance cannot be saturated or self-detuned, which means the fourfold structure will be maintained as long as the beam evolves.

\section{DISCUSSIONS AND CONCLUSIONS}

The problems of envelope instability as a short term effect and the fourth order of resonance as a long term effect, due to nonlinear space charge, are both discussed in this paper. In the parameter region where both resonances are excited, the results from the self-consistent code TOPOPIC depict both effects respectively. The observed stop band corresponds to envelope instability (or the second even mode). Moreover, the sharp falling effect of emittance growth behavior is also perfectly explained.

It should be noted that the envelope instability is rms mismatch dependent. The effect of the envelope instability can actually be minimized in theory by nearly perfectly rms envelope matching. In addition, this envelope instability is considered as a core effect, which means this will hardly result in beam halos. In another case, with the same mismatch condition, the time scale of saturation $\tau$ for equivalent rms matched but different initial distributions (except the KV beam) is supposed to be in the same order of range. Definitely, the relationship between emittance growth and saturation time $\tau$ from nonlinear resonance is still a big subject and merits further careful studies.

\section{ACKNOWLEDGMENTS}

One of the authors, C. L., extends his sincere thanks to R.A. Jameson and Y.K. Batygin for meaningful suggestions and discussion. This work is supported by the Ministry of Science and Technology of China under Grant No. Y41J22207A.

[1] J. Stovall, J. Billen, S. Nath, H. Takeda, L. Young, D. Jeon, K. R. Crandall, and R. Shafer, in Proceedings of the 19th Particle Accelerator Conference, Chicago, IL, 2001 (IEEE, Piscataway, NJ, 2001), Vol. 1, pp. 446-450.

[2] Y. Yamazaki, Proceedings of the 2003 Particle Accelerator Conference, Portland, 2003, C030512, 576 (IEEE, Portland, 2003).

[3] Z. Li, P. Cheng, H. Geng, Z. Guo, Y. He, C. Meng, H. Ouyang, S. Pei, B. Sun, J. Sun, J. Tang, F. Yan, Y. Yang, C. Zhang, and Z. Yang, Phys. Rev. ST Accel. Beams 16, 080101 (2013).

[4] B. V. Chirikov, Phys. Rep. 52, 263 (1979).

[5] J. M. Lagniel and D. Libault, in Proceedings of the Particle Accelerator Conference, Dallas, TX, 1995 (IEEE, New York, 1995), Vol. 5, pp. 3235-3237.

[6] T. P. Wangler, K. R. Crandall, R. Ryne, and T. S. Wang, Phys. Rev. ST Accel. Beams 1, 084201 (1998).

[7] J. Struckmeier and M. Reiser, Part. Accel. 14, 227 (1984).

[8] I. Hofmann, L. J. Laslett, L. Smith, and I. Haber, Part. Accel. 13, 145 (1983).

[9] R. L. Gluckstern, Proceedings of the 1970 Proton Linear Accelerator Conference, Batavia, 1970, C700928, 811 (Batavia, 1970), http://www.osti.gov/scitech/biblio/4619173.

[10] I. Hofmann, Phys. Rev. E 57, 4713 (1998).

[11] R. A. Jameson, Self-Consistent Beam Halo Studies \& Halo Diagnostic Development in a Continuous Linear Focusing Channel (World Scientific, Singapore, 1994), pp. 530-560.

[12] M. Tabor, Chaos and Integrability in Nonlinear Dynamics: An Introduction (Wiley, New York, 1989).

[13] R. A. Jameson, in Proceedings of the 1981 Linear Accelerator Conference, Santa Fe, NM, 1981 (unpublished).

[14] D. Jeon, L. Groening, and G. Franchetti, Phys. Rev. ST Accel. Beams 12, 054204 (2009).

[15] L. Groening, W. Barth, W. Bayer, G. Clemente, L. Dahl, P. Forck, P. Gerhard, I. Hofmann, M. S. Kaiser, M. Maier, S. Mickat, T. Milosic, D. Jeon, and D. Uriot, Phys. Rev. Lett. 102, 234801 (2009).

[16] V. I. Arnold, Mathematical Methods of Classical Mechanics, 2nd ed. (Springer, New York, 1997).

[17] C. Li, Chin. Phys. C 38, 037005 (2014).

[18] C. Li, Nucl. Instrum. Methods Phys. Res., Sect. A 770, 169 (2015).

[19] http://irfu.cea.fr/Sacm/logiciels/index3.php.

[20] G. Franchetti and I. Hofmann, Nucl. Instrum. Methods Phys. Res., Sect. A 561, 195 (2006). 